\title{
Multiple shock waves in the atmosphere of the Cepheid X Sagittarii?
}

\author{
P. Mathias ${ }^{1}$, D. Gillet ${ }^{2}$, A. B. Fokin ${ }^{1}$, N. Nardetto ${ }^{1}$, P. Kervella $^{3}$, and D. Mourard ${ }^{1}$ \\ 1 Observatoire de la Côte d'Azur, GEMINI, UMR 6203, BP 4229, 06304 Nice Cedex 4, France \\ 2 Observatoire de Haute Provence, 04870 Saint-Michel l'Observatoire, France \\ 3 Observatoire de Paris-Meudon, LESIA, UMR 8109, 5 place Jules Janssen, 92195 Meudon Cedex, France
}

Received 28 March 2006 / Accepted 5 June 2006

ABSTRACT

\begin{abstract}
Context. Shock waves in Cepheids have often been invoked, both from observational and theoretical points of view. However, classical shock wave signatures, such as emission or line doubling, have hardly been detected.

Aims. In this paper, we suggest that our spectra of the classical Cepheid X Sgr can be interpreted by means of the passage of 2 shock waves per pulsation period.

Methods. We study new, high-resolution (120000) spectra of X Sgr that show very complicated patterns within metallic lines.

Results. Spectra show up to 3 components in most of the lines of the spectra during most of the pulsation cycle. These components seem to follow a pulsation motion. In the blue wing, the appearance of a new component is observed twice per pulsation period, which can be interpreted by 2 consecutive shock waves, one being apparently related to the classical $\kappa$-mechanism at work in these stars. The origin of the second shock is still unclear.

Conclusions. X Sgr is an exceptional Cepheid according to its observed multi-components behaviour. Additional observations are requested in order to establish the eventual effect of the binary orbit on the pulsation motion.
\end{abstract}

Key words. techniques: spectroscopic - stars: individual: X Sgr - stars: variables: Cepheids - stars: oscillations stars: binaries: general

\section{Introduction}

Generally, line profile variations, including those in classical Cepheids, reflect the motions of the gas in a radially pulsating atmosphere. Such behaviour is now well understood in the framework of radiative hydrodynamics (see e.g. Fokin et al. 1996). However, some Cepheids were reported as demonstrating unusual behaviour in their line profiles.

Evidence of line doubling in a number of low-excitation transitions was first reported by Kraft (1956) for X Cyg. Kraft (1967) has shown that line doubling in this star was varying from cycle to cycle, whereas Butler (1993) did not recover this effect. Sasselov et al. (1989) and Sasselov \& Lester (1990) noticed line doubling (splitting) in X Sgr infrared spectra. The authors also point out the stability of this phenomenon during 11 months.

Kovtyukh \& Andrievsky (1999) detected anomalous splitting in the spectrum of EV Sct and explain it as a sign of spectroscopic binarity, but the dynamics of the spectra over 10 years does not favour this hypothesis. Kovtyukh et al. (2003) observed features in the line profiles of X Sgr, EV Sct, BG Gru, and V1334 Cyg, 3 of them s-Cepheids, while X Sgr may also belong to this group of first-overtone pulsators, characterised by smallamplitude and sinusoidal light curves. These authors interpret the presence of observed bumps as the combined effect of strong line broadening (rotation, macroturbulence) and non-radial oscillations, excited through resonances.

The aim of this paper is to test another interpretation of the line-profile variations of X Sgr in terms of shock waves. Our new high spectral-resolution data are described in Sect. 2. Section 3 deals with the description of the line doubling phenomenon. An interpretation of our data, involving shock waves, is provided in Sect. 4, while different hypotheses are discussed in Sect. 5. Finally, some concluding remarks are given in Sect. 6.

\section{Observations and data reduction}

All the spectra discussed in this paper have been obtained with the HARPS spectrograph attached to the $3.6 \mathrm{~m}$ telescope located at La Silla (ESO). A total of 24 spectra (resolving power of $R=120000$ ) were obtained from February 20 to March 2, 2004, covering $120 \%$ of a pulsation cycle. However, because most of the spectra were obtained as very close consecutive pairs, only 11 different dates are considered in this paper. Reduction processes have already been described in Nardetto et al. (2006b).

In the following, we concentrate mainly on a metallic line free of blend: Fe I $\lambda \lambda$ 6056. All pulsation phases were computed using the ephemeris provided by Berdnikov \& Caldwell (2001): $P=7.01281 \mathrm{~d}$ and $T_{0}=2451653.56 \mathrm{~d}$. Pulsation phases corresponding to the observation dates are given in Table 1 .

\section{Appearance of 2-3 components}

Figure 1 represents the evolution of the considered Fe I line profile. It is clear that at least 2 components are simultaneously present, and most of the time, 3 components show up. This particular structure is easily detected in all metallic lines, as shown in Fig. 2. Therefore, the behaviour discussed for Fe I $\lambda \lambda 6056$ can be generalized to all metallic lines.

Using a least-square procedure involving 3 Gaussians, we tried to track the evolution of each component (Fig. 1). Most of 
Table 1. Logbook of observations (February 20 - March 2, 2004). Julian Dates $(-2453000)$ and corresponding pulsation phase are given (see text).

\begin{tabular}{cc}
\hline \hline JD & $\varphi$ \\
\hline 55.9004 & 0.9684 \\
55.9030 & 0.9688 \\
56.8980 & 1.1107 \\
56.9010 & 1.1111 \\
56.9045 & 1.1116 \\
56.9075 & 1.1120 \\
57.8992 & 1.2534 \\
57.9026 & 1.2539 \\
58.8994 & 1.3960 \\
58.9032 & 1.3966 \\
59.9017 & 1.5390 \\
59.9057 & 1.5395 \\
60.8981 & 1.6811 \\
60.9012 & 1.6815 \\
61.8967 & 1.8234 \\
61.8991 & 1.8238 \\
62.8947 & 1.9658 \\
62.8973 & 1.9661 \\
63.9013 & 2.1093 \\
63.9043 & 2.1097 \\
64.9015 & 2.2519 \\
64.9050 & 2.2524 \\
66.8960 & 2.5363 \\
66.9000 & 2.5369 \\
\hline
\end{tabular}

the time, all components are regularly moving from blue to red, as individual shells would do following decelerating motions, hypothetically after a shock passage. Figure 3 represents the radial velocities associated to each component of Fig. 1. There seems to be a periodicity for all these curves. We also note that line profiles associated to the same phase of different pulsation cycles are not completely similar: there is no reproductibility of the pulsation motion from cycle-to-cycle.

This particular behaviour, unique among the classical Cepheids, affect all weak lines of the spectra, but marginally affect the relatively strong lines such as the $\mathrm{NaD}$ doublet or the Ba II $\lambda \lambda 4934$ line. In addition, Balmer lines do not present this behaviour. Figure 4 represents the evolution of the $\mathrm{H} \alpha$ profile. A rapid velocity shift is observed between $\varphi=1.823$ and $\varphi=1.966$, but no component can be detected related to the pulsation. However, it seems possible that the intrinsically large widths of these strong lines (1.6-2.5 $\AA$ ) can hide the presence of an eventual line doubling, which has a typical Doppler shift of about $0.8 \AA$.

From Fig. 3, we can see that the complicated behaviour of the observed absorption components can be represented by a -nearly- periodic system of 2 fragments of velocity curves, repeated from cycle to cycle and overlapping for most of the time. It is interesting to note that the Doppler shift between them is always of the order of $20-35 \mathrm{~km} \mathrm{~s}^{-1}$ i.e., a value corresponding to a typical radial velocity amplitude of Cepheids.

Whereas Sasselov \& Lester (1990) mention the presence of 2 components in their infrared spectra, a careful inspection of their Fig. 12 also reveals that 3 components can be detected in the line profiles obtained in 1989. Therefore, within the past 15 years, the appearance of 2 or 3 components is variable. From these observations, a single profile has never been observed, in contrast to the usual spectra of classical Cepheid stars.
HD 161592 FeI 6056 A

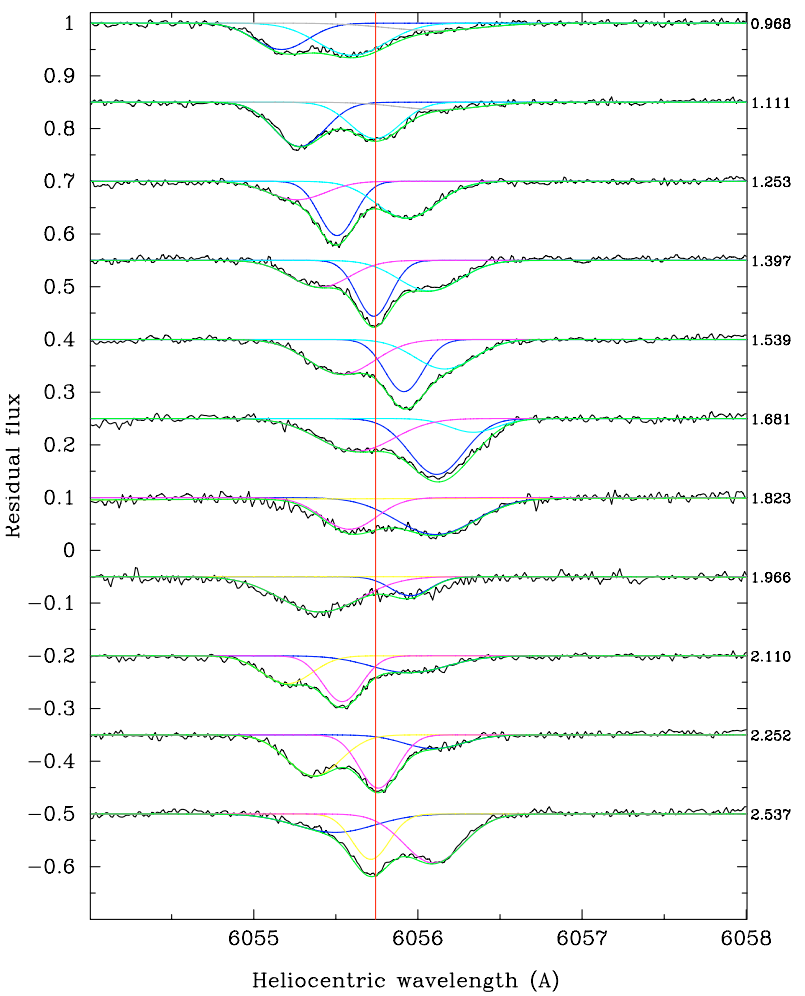

Fig. 1. Evolution of the Fe I $\lambda \lambda 6056$ line profile. On the right are the pulsation phases. The Gaussian fits corresponding to the different components are indicated, the evolution of each component being symbolised by a given colour. The red vertical line represents the rest wavelentgh.

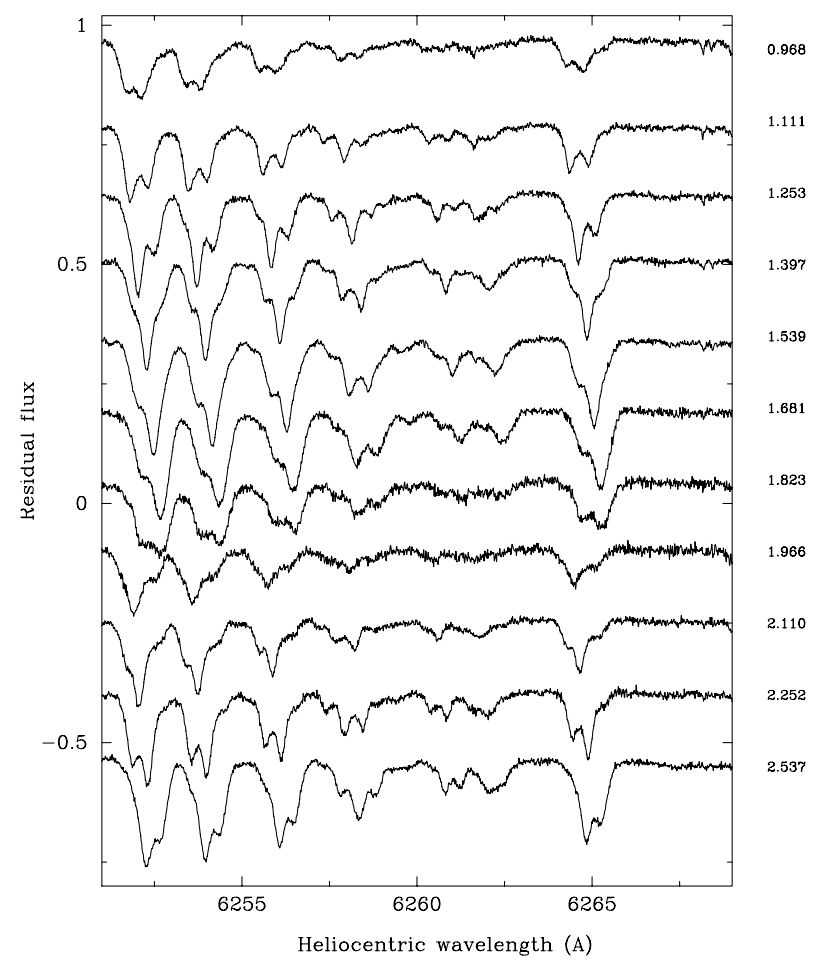

Fig. 2. Evolution of the different line profiles for the same phases shown in Fig. 1. From blue to red, the presented lines are Fe I (6252 $\AA$ ), Si I (6254 ̊̊), Fe I (6256 ̊), Ti I (6258 ̊), unidentified, and Fe I $(6265 \AA)$. 


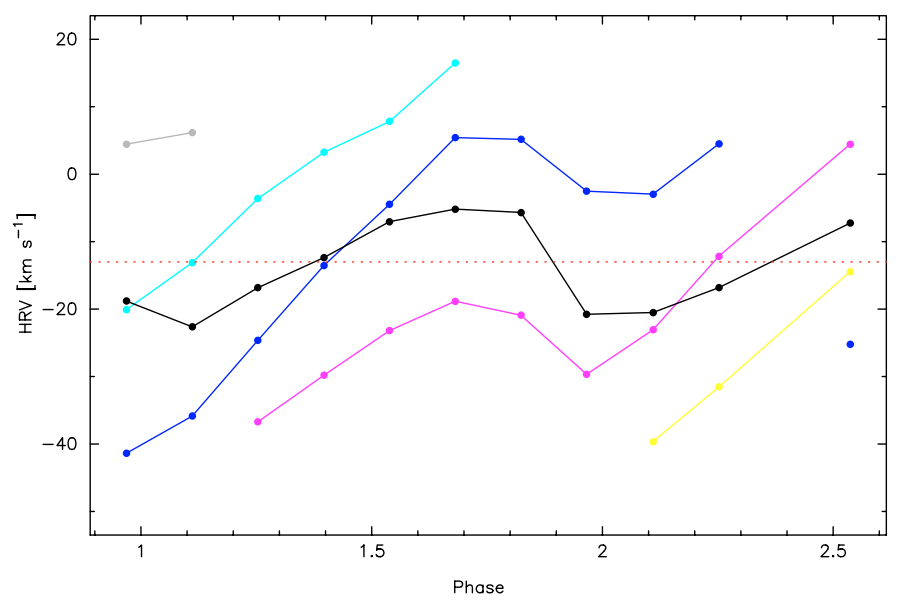

Fig. 3. Radial velocities associated to each component measured in Fig. 1. The same colour code has been used. Black dots represent the velocity associated to the centroid of the whole profile. The horizontal red line represents the velocity of the star in the heliocentric frame.

\section{Two shocks per pulsation period?}

The most prominent feature in our high-resolution spectra is thus the appearance of 2-3 well-separated line components. However, the velocity associated with the centroid of the whole line profile behaves, except for the amplitude, as a classical Cepheid radial velocity curve (see e.g. Nardetto et al. 2006b). This suggests that all components are directly linked to the pulsation (Fig. 3).

Such line-splitting phenomena can be associated to atmospheric dynamics. Indeed, line doubling is often observed when a shock wave propagates outwards in the atmosphere of largeamplitude pulsating stars, following the Schwarzschild's mechanism (1952). The behaviour of the 3 components observed in Figs. 1 and 2 can indeed be interpreted by means of the propagation of 2 shock waves per pulsation period.

At $\varphi=0.968$, a shock (hereafter shock 1) appears near the photosphere and starts to propagate upwards. The Schwarzschild mechanism is clearly observed at $\varphi=1.110$, where the bluest component increases in strengh compared to the other one. This phenomenon is also observed at $\varphi=1.966,2.110$, except that the doubling is not resolved as well. Therefore, as already observed in other stars undergoing strong non linear motions (see e.g. Fokin et al. 2004), irregularities are present in the shock intensity, due to an atmospheric time relaxation that is longer than the pulsation period. Thus, the shock is not self similar from one cycle to the next. This shock coincides with the rapid blueshift of the $\mathrm{H} \alpha$ line (Fig. 4) and, seemingly, corresponds to the main "engine" of the pulsation.

In addition to this phenomenon, a second shock (hereafter shock 2 ) is present at $\varphi=1.253$ and $\varphi=2.537$, occurring during the beginning of the fall of the upper atmosphere. Since the consecutive components are separated by the same quantity, $\Delta V \approx 30 \mathrm{~km} \mathrm{~s}^{-1}$, this shock has an intensity that is comparable to the main one. Again, due to the complicated motion of the atmosphere, irregularities are present. Indeed, the occurrence of shock 2 differs by more than $0.25 P$ from one cycle to another.

\section{Origin of the third component}

Seemingly, shock 1 should be driven by the $\kappa$-mechanism usually at work in these stars, due to the helium and hydrogen ionization zones. However, note that no known classical

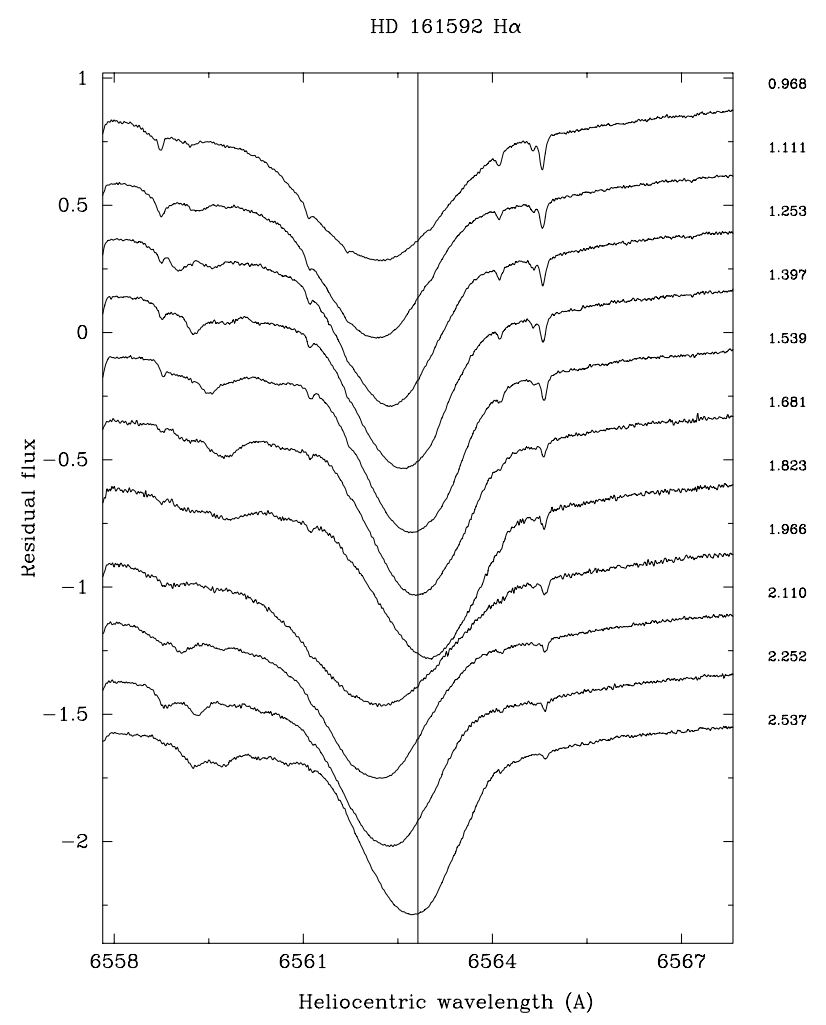

Fig. 4. Same as Fig. 1, but for the $\mathrm{H} \alpha$ line.

Cepheids show a line-doubling phenomenon associated to this usual $\kappa$-mechanism, which is the consequence of the propagation of a strong shock wave.

The origin of shock 2 is still unclear. While 2 shocks per pulsation periods are not very rare (Fokin et al. 2004 for $\beta$ Cephei stars, Fokin 1992 for RR Lyrae stars), such a phenomenon has not been reported yet for classical Cepheid stars. In addition, the already detected second shock in the $\beta$ Cephei star BW Vul and in RR Lyrae stars occurs normally during the stellar contraction, not during the expansion. In the case of the $\beta$ Cephei star BW Vulpeculae, while the main shock is due to the iron ionization zone, the second shock is due to another bump mechanism in a different ionization zone.

A detailed study of the work integral along the stellar envelopes is required to test this hypothesis further, in order to understand why X Sgr has such peculiar behaviour compared to Cepheids of a similar pulsation period, such as $\eta$ Aql, which presents classical line-profile variations (Kiss \& Vinkó 2000). In addition, up to now, no pulsation model has been able to provide a double shock wave within a unique pulsation cycle.

It is believable that an "external" phenomenon i.e., one not linked to the pulsation, acts to perturb the classical pulsation model to explain the presence of the 2-3 line components and the presence of a second shock. Hereafter, we discuss whether the activity/Non Radial Pulsations (NRP) or an SB2 (or even an SB3?) phenomenon can be related to the generation of the line components.

\subsection{The activity/NRP hypothesis}

It does not seem possible that the appearance of the different components is provoked by some rotating structures or objects, like clouds. Indeed, the rotation velocity would be more than $200 \mathrm{~km} \mathrm{~s}^{-1}$, considering an equatorial velocity of $10 \mathrm{~km} \mathrm{~s}^{-1}$. 
Activity, such as hot photospheric spots or convection, can affect the spectrum. However, in order to produce such large components, convective cells should be very large, of the order of the stellar radius. This phenomenon can be expected in cool stars, not in F-stars.

Kovtyukh et al. (2003) suggested that the line profile variations is due to NRP. This interpretation is plausible, but not free of difficulty. The amplitude of such non-radial pulsation must be of the order of the total observed Doppler shift between the 3 components (about $40 \mathrm{~km} \mathrm{~s}^{-1}$ ), which seems too high. Indeed, since the sound-speed velocity is about $10 \mathrm{~km} \mathrm{~s}^{-1}$ in Cepheids (e.g. Gillet et al. 1999), the question arises as to how different parts of the star can be physically connected as required in the NRP hypothesis: the linear solution cannot be applicable. Also, it is not clear how such non-radial pulsation can co-exist with the radial one and why their periods are very much alike.

\subsection{The binary hypothesis}

Using all available spectroscopic data from the beginning of the $20^{\text {th }}$ century, Szabados (1990) computed the $\gamma$-velocities and proposed that X Sgr was the main component of a binary system having an orbital period of $507 \mathrm{~d}$.

Therefore, the different components could be due to the different members of a multible system, or else due to another Cepheid with a similar mass in order to have a similar pulsation period. However, both hypotheses seem unlikely, since the periods cannot be identical and a kind of beat-period would have already been revealed in long-term studies, both in photometry and in spectroscopy, whereas no such phenomenon has ever been reported. Moreover, X Sgr is not known to have a photometric companion (Evans 1992). The companion could be a compact object. Indeed, using the orbital elements of Szabados (1990) and a mass of $4.5 M_{\odot}$ (Caputo et al. 2005), this projected companion mass is of the order of $0.2-0.3 M_{\odot}$.

From this orbital period, it is interesting to compute the mean orbital phase $\Psi$ corresponding to the most recent highresolution spectroscopic observations, i.e., those of Sasselov \& Lester (1990) obtained in 1988 and 1989, in addition to our observations. Arbitrarily setting the orbital phase of the April 1988 data to $\Psi=0$, the 1989 and 2004 phases have been obtained at $\Psi=0.46$ and $\Psi=0.41$, respectively. Thus, the 1989 and 2004 data have been obtained at a similar orbital phase. A careful inspection of Fig. 12 in Sasselov \& Lester (1990) reveals that 3 components can be detected in the line profiles obtained in 1989, while only 2 components seem to be present in 1988 .

This coincidence could suggest a possible link between the binary motion and the appearance of a third component. The influence of tidal effects affecting the line shape has already been discussed in Moreno et al. (2005), but the two cases are very different: the stars are relatively close to each other and the eccentricity is high.

Unfortunately, no high-resolution spectra are available in the literature between 1989 and 2004, hence no estimate on the time scale of the presence of the 3 components can be derived. In addition, the above coincidence is based only on the value of the period provided by Szabados (1990). The value of this orbital period, $507.25 \mathrm{~d}$, is based on a fit of $\gamma$-velocities. However, if we add our $\gamma$-velocity value, $-12.7 \mathrm{~km} \mathrm{~s}^{-1}$, this last point does not fit the supposed variation at all, as shown in Fig. 5. We also computed the Fourier spectrum of these $\gamma$-velocities, but it appears that many peaks are present, a "dominant" peak being hardly detected at a period $575 \mathrm{~d}$, slightly higher than the $507 \mathrm{~d}$ value. This noisy spectrum is the consequence of the large uncertainties

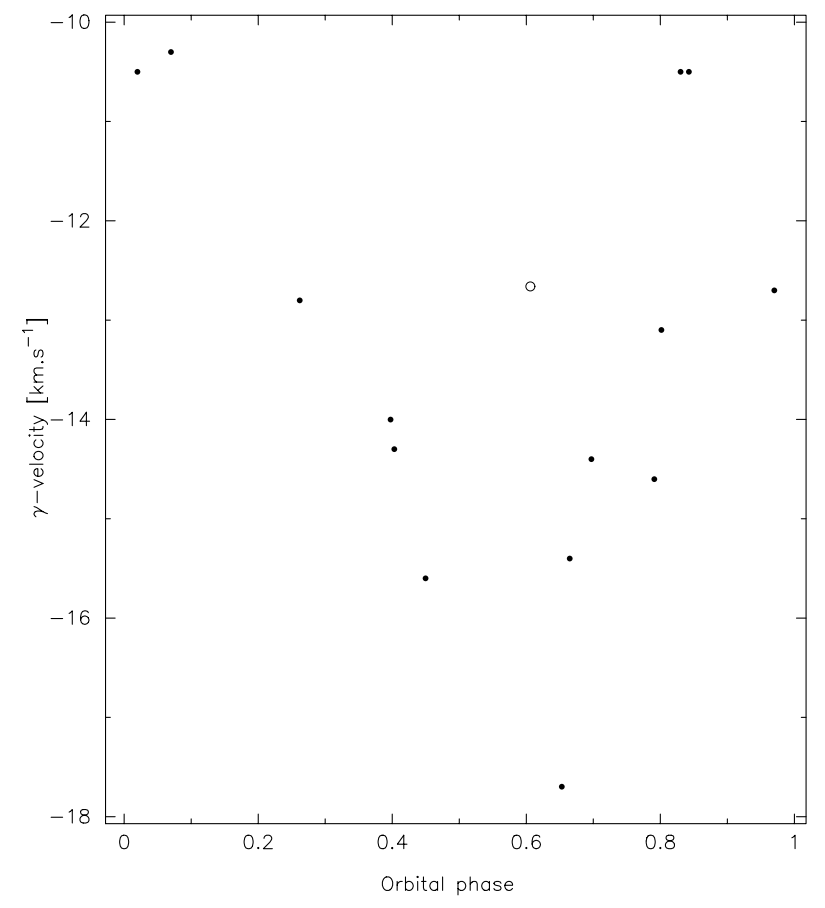

Fig. 5. $\gamma$-velocities taken from Szabados (1990) [points] and our new $\gamma$-velocity value [circle] phased with the orbital period 507.25.

associated to the considered $\gamma$-velocities, which can result from very old observations and the merging of different cycles. These uncertainties are approximately the amplitude of the orbital motion $\left(2 \mathrm{~km} \mathrm{~s}^{-1}\right)$. Using the period $575 \mathrm{~d}$, the orbital phases associated to the 1988,1989 , and 2004 observations are $\Psi=0,0.40$, and 0.05 , respectively: the situation is completely different to the scenario discussed in the preceding paragraph.

Therefore, before going further into the possible role of the binarity in the pulsation motions, a fundamental step is to confirm the binary system status of X Sgr, and then to compute the orbital elements.

\section{Discussion and conclusion}

These multiple line components have never been reported before, except in Sasselov et al. (1989) and Sasselov \& Lester (1990) who mention only 2 components, whereas 3 components are present in their spectra obtained in 1989. Kovtyukh et al. (2003) interpret travelling bumps in terms of non radial pulsations. An inspection of their Figs. 4 and 5 indeed shows the presence of different components; especially, in their Fig. 4, the spectra at phases 1.966 and 1.228 are similar to ours at phase 1.397. Thus, the multiple line-component interpretation is also possible in these cases. Kovtyukh et al. (2003) also mention that spectra obtained by Luck \& Lambert $(1981,1985)$ in 1978-1979 present absorption bumps.

Therefore, the presence of these multiple components may be related to a particular evolutionary status. Indeed, as mentioned by Kovtyukh et al. (2003), X Sgr may be in a unique evolutionary stage. However, from this point of view, X Sgr presents a rather modest period increase $\left(\log \mathrm{d} P / \mathrm{d} T=+0.172 \mathrm{sec}^{\mathrm{year}}{ }^{-1}\right.$ Turner 1998) compared to other cases. It seems therefore unrealistic that such a phenomenon could be attributed to evolutionary internal structure rearrangement.

Our observations suggest the propagation of 1 or 2 shock waves per pulsation period. While one can be related to the usual 
$\kappa$-mechanism acting in these stars, the origin of the second shock is still unclear and could be related to the physical conditions in the envelope that could favour a second $\kappa$-mechanism in another ionization zone. This second destabilisation mechanism can also be caused by external forces, such as tidal effects. As the binarity of the star is still a hypothesis, however, it is important to observe the star again with the aim of definitively establishing or ruling out the presence of a companion.

Even though the observational data formally fit the Schwarzschild mechanism and, thus, the shock hypothesis well, several questions arise:

1. If this effect is intrinsic to the star, why has it never been observed in other Cepheids?

2. In all earlier Cepheid studies, the shocks in the metal Line Forming Region (LFR) have negligeable amplitude, in strong contrast to X Sgr. According to the models, the compression waves, or weak shocks, normally also pass through the LFR in several hundredth of the period, since this zone is geometrically very thin. In X Sgr, the hypothetical shocks should be very "slow" and pass the LFR in about $50-70 \%$ of the pulsation period. However, because clear line doubling is detected, the shock should be stronger than in other classical Cepheids, so the shock front should be fast. A possible way to reconcile these points of view is to consider a large enhancement of the atmospheric extension due to, for instance, tidal effects. Following the theory of stellar shocks, the outermost atmosphere must fall in a ballistic way with a characteristic time approximately $150-170 \%$ of that of the photospheric layers. A simplified estimation shows that $t_{\text {out }} / t_{\text {phot }} \approx\left(R_{\text {out }} / R_{\text {phot }}\right)^{1.5}$, which gives $R_{\text {out }} / R_{\text {phot }} \approx 1.3-1.4$.

3. If the atmosphere is so extended, why is there no phase lag between weak metal lines, forming low in the amosphere, and $\mathrm{H} \alpha$, which is formed higher? Such a phase lag has indeed been observed in the $\beta$ Cephei star BW Vulpeculae (Mathias et al. 1998).

Therefore, the origin of the multiple line components in X Sgr is still a mystery, so this star should be observed continuously in order to determine its pulsation characteristics better. In addition, using the value of the limb-darkened angular diameter of X Sgr, $1.471 \pm 0.033$ mas (Kervella et al. 2004), we plan to observe the star with the AMBER instrument of the VLTI in the high spectral-resolution mode. The idea would be to follow each component in order to provide a $3 \mathrm{D}$ view of the stellar atmosphere and to determine if the associated shells are physically separated by our hypothetical shock fronts. Indeed, using theroretical intensity maps, we have shown (Nardetto et al. 2006a) that the location of the LFR can be followed throughout the pulsation period.

Acknowledgements. This work was based on observations collected at La Silla Observatory, Chile, in the framework of European Southern Observatory's programme ID 072.D-0419. The authors thank the anonymous referee who provided many useful suggestions for improvements.

\section{References}

Berdnikov, L. N., \& Caldwell, J. A. R. 2001, J. Astron. Data, 7

Butler, R. P. 1993, ApJ, 415, 323

Caputo, F., Bono, G., Fiorentino, G., Marconi, M., \& Musella, I. 2005, ApJ, 629, 1021

Evans, N. R. 1992, ApJ, 384, 220

Fokin, A. B. 1992, MNRAS, 256, 26

Fokin, A. B., Gillet, D., \& Breitfellner, M. G. 1996, A\&A, 307, 503

Fokin, A., Mathias, P., Chapellier, E., Gillet, D., \& Nardetto, N. 2004, A\&A, 426, 687

Gillet, D., Fokin A. B., Breitfellner, M. G., Mazauric, S., \& Nicolas, A. 1999, A\&A, 344, 935

Kervella, P., Nardetto, N., Bersier, D., Mourard, D., \& Coudé du Foresto, V. 2004, A\&A, 416, 941

Kiss, L. L., \& Vinkó, J. 2000, MNRAS, 314, 420

Kovtyukh, V. V., \& Andrievsky, S. M. 1999, A\&A, 350, L55

Kovtyukh, V. V., Andrievsky, S. M., Luck, R. E., \& Gorlova, N. I. 2003, A\&A, 401,661

Kraft, R. P. 1956, PASP, 68, 137

Kraft, R. P. 1967, in Aerodynamic Phenomena in Stellar Atmospheres, ed. R. N. Thomas (New York), IAU 28, 207

Luck, R. E., \& Lambert, D. L. 1981, ApJ, 245, 1018

Luck, R. E., \& Lambert, D. L. 1981, ApJ, 298, 782

Mathias, P., Gillet, D., Fokin, A. B., \& Cambon, T. 1998, A\&A, 339, 525

Moreno, E., Koenigsberger, G., \& Toledano, O. 2005, A\&A, 437, 641

Nardetto, N., Fokin, A. B., Mourard D., \& Mathias, P. 2006a, A\&A, 454, 327

Nardetto, N., Mourard, D., Kervella, P., et al. 2006b, A\&A, 453, 309

Sasselov, D. D., Lester, J. B., \& Fieldus, M. S. 1989, ApJ, 337, L29

Sasselov, D. D., \& Lester, J. B. 1990, ApJ, 362, 333

Schwarzschild, M. 1952, Transactions of the IAU VIII, ed. P. Th. Oosterhoff (Cambridge: Cambridge Univ. Press), 811

Szabados, L. 1990, MNRAS, 242, 285

Turner, D. G. 1998, JAAVSO 26 